\title{
Situation-evoking stimuli, domain of reference, and the incremental interpretation of lexical ambiguity
}

\author{
HOANG VU \\ Saint Mary's College of California, Moraga, California \\ and \\ GEORGE KELLAS, ERIC PETERSEN, and KIM METCALF \\ University of Kansas, Lawrence, Kansas
}

\begin{abstract}
In two experiments, we examined the influence of situation-evoking stimuli on the resolution of lexical ambiguity. In Experiment 1, we examined situation-evoking stimuli at an early NP position. Readers were asked to establish whether specific entities were likely to participate as agents in contextually defined situations. Naming latencies demonstrated that defined situations headed by likely agents evoked a domain of reference that included only the situation-appropriate meaning of a targeted lexical ambiguity. In contrast, defined situations headed by unlikely agents evoked a domain of reference that did not include either meaning of the intended ambiguous word. In Experiment 2, we examined situation-evoking stimuli at a later direct object position. The specificity of the theme/patient role filler was manipulated, where the linguistic expressions were either specific or general with respect to a given contextual situation. The results showed that contexts with specific situation-evoking stimuli were rated as strongly biased and provided a domain of reference for the immediate resolution of lexical ambiguity, whereas contexts with nonspecific role fillers were rated as ambiguous and provided a domain of reference that was supportive of both meanings of an ambiguous word. The results were discussed within a contextual-feature-sensitive model of language processing.
\end{abstract}

When a reader processes a sentence, several levels of analysis are required for comprehension, including, but not limited to, recognition of the perceptual input, retrieval of individual word meanings, parsing for grammatical structure, assignment of thematic roles, and drawing inferences on the basis of general world knowledge. The reader computes and integrates this information quickly and easily, yet the actual processes that govern language comprehension are still elusive, despite decades of research. One influential approach proposes that comprehension is achieved, in part, via the activation of stereotypical precompiled structures of knowledge, such as scripts and schemas (e.g., Bower, Black, \& Turner, 1979; Schank \& Abelson, 1977), or of more general situations (cf. Ferretti, McRae, \& Hatherell, 2001; Sanford \& Garrod, 1981; Traxler, Foss, Seely, Kaup, \& Morris, 2000; Zwaan \& Radvansky, 1998). According to more formal script/schema theories, understanding requires the activation of a script, using script headers (e.g., a restaurant) and then the subsequent "filling in" of the slots of the script

This research was supported in part by grants from the Faculty Development Fund of Saint Mary's College, awarded to the first author. We thank Lee Osterhout, Matthew Traxler, and two anonymous reviewers for their helpful comments. Correspondence concerning this article should be addressed to H. Vu, Department of Psychology, Saint Mary's College, 1928 Saint Mary's Road, Moraga, CA 94575 (e-mail: hvu@stmarys-ca.edu). (e.g., agents, patients, instruments, etc.) on the basis of information available from the discourse. However, the rigid structure of scripts exposes several problems for such proposals, such as the level of detail of a stored script, how much information is activated when a script is instantiated, and how comprehension progresses when subsequent information has no apparent relation to a script (see Bower et al., 1979; Whitney, Budd, Bramucci, \& Crane, 1995).

A more flexible alternative views language comprehension as an incremental process of constraint satisfaction (e.g., Taraban \& McClelland, 1988), in which there is a dynamic interaction between the streaming linguistic input and the activation of generalized situations. On this view, comprehension is achieved via an incremental word-by-word construction of a situational representation of the discourse context - a running discourse model of comprehension that is continuously updated, revised, and refined to satisfy the accumulating constraints imposed by the text and general world knowledge (e.g., Sanford \& Garrod, 1981; see also Johnson-Laird, 1983). ${ }^{1}$ A linguistic stream will tap a general situation whenever there is enough available information to do so, and the situation that is invoked will contain information corresponding to the linguistic input itself, as well as other information specific to the situation. The evoked situation provides a domain of reference for interpreting subsequent information, yet simultaneously, each new lexical 
entry will incrementally update the situational model and, thus, refine the domain of reference (e.g., Altmann \& Kamide, 1999; Schwanenflugel \& Shoben, 1985; Sedivy, Tanenhaus, Chambers, \& Carlson, 1999).

When the situational model is updated, the domain of reference may be maintained, elaborated, and refined or may be completely revised, depending on the compatibility of incoming lexical information (see Zwaan \& Radvansky, 1998). Consider, for example, the following sentence frames:

1a. The astronomer knew the name of the

1b. The astronomer liked the flavor of the

In both sentences, it is likely that when the agent "astronomer" is processed, a situational-levelrepresentation is triggered, with features associated with astronomer being computed, such as $<$ stars $><$ space $><$ moon $><$ etc. $>$, as part of its domain of reference. When "knew the name of the" is processed in 1a, features such as <understand $>$ $<$ possess knowledge $><$ identity $>$ are mapped onto the semantic representation. The semantic trajectory of the evolving reference domain of 1 a remains on course, and readers will expect referring expressions that are related to an astronomer (e.g., star) to complete the sentence. However, in $1 \mathrm{~b}$, the reference domain must be substantially revised in order to incorporate referents that will be compatible with the modifier "flavor." It is extremely unlikely that readers would expect a linguistic expression that is related specifically to an astronomer to complete the sentence. Thus, although an activated situation provides a reference domain for the interpretation of subsequent linguistic information, the fate of the evolving situational representation is determined by the actual linguistic input. The appropriate situation model of the context will be derived through continuous refinements and revisions via an incremental process of constraint satisfaction.

The importance of situation-based knowledge for lexical processing has been demonstrated by McRae, Hare, Ferretti, and Elman (2001), who showed that discourse entities (agents, patients, instruments) primed verbs via event schemas. It was proposed that generalized events are organized in such a way that the participants in an event can be used to activate the event structure and, consequently, to activate the verb that is typically associated with the event. In addition, Hess, Foss, and Carroll (1995) found that the speed of processing of a sentence-final word depended more on the fit of the word with the overall situational context than on lexical associates immediately prior to the final word. Finally, situational knowledge can be used to interpret locally neutral and nonspecific information (see Sanford \& Garrod, 1981).

\section{Situation-Based Knowledge and the Resolution of Lexical Ambiguity}

An important tool used to study language comprehension is lexical ambiguity, or words with multiple distinct meanings (e.g., a mammal bat vs. a baseball bat). How ambiguous words are resolved in context is a nontrivial question, since an understanding of this issue can provide answers to fundamental questions regarding the architecture of the language comprehension system and the roles that discourse context and general world knowledge play during language processing. The early seminal research of Swinney (1979) and Seidenberg, Tanenhaus, Leiman, and Bienkowski (1982) supported a language system that is modular in architecture (Fodor, 1983), in which the immediate activation of word meanings proceeds without influence from discourse context and general world knowledge. This early evidence was contradicted by later research (e.g., Paul, Kellas, Martin, \& Clark, 1992; Simpson \& Krueger, 1991; Van Petten \& Kutas, 1987), which showed that context can have an immediate influence on ambiguity resolution, thus supporting an interactive-activation viewpoint (e.g., McClelland, 1987) in which discourse context and world knowledge can immediately influence the resolution of lexical ambiguity. More recent research has suggested that the resolution of lexical ambiguity depends on the factors of meaning frequency, type of context used, and context strength. For example, Tabossi, Colombo, and Job (1987) and Paul et al. (1992) have demonstrated that feature-denoting contexts could be used to prime probe words representing those features of ambiguous words. And in a series of studies, Rayner and colleagues (e.g., Dopkins, Morris, \& Rayner, 1992; Duffy, Morris, \& Rayner, 1988; Rayner, Pacht, \& Duffy, 1994) have shown that an intricate relationship exists between meaning frequency and biasing context in lexical ambiguity resolution (see also Hogaboam \& Perfetti, 1975). Briefly, these researchers have shown that lexical activation is ordered by meaning frequency but that a biasing context can reorder the availability of the various meanings. However, they argued that context cannot override the influence of meaning frequency, or minimally does so (e.g., Wiley \& Rayner, 2000). In contrast, Simpson and Krueger (1991) have demonstrated that a strongly biased context could lead to the activation of only the contextually appropriate meaning of an ambiguous word. Finally, Kellas and colleagues (e.g., Martin, Vu, Kellas, \& Metcalf, 1999; Vu, Kellas, Metcalf, \& Herman, 2000; Vu, Kellas, \& Paul, 1998) have established that ambiguity resolution depends on a complex interaction among meaning frequency, context type, and strength of the biased context. Critically, it was found that although meaning frequency does play an important role, its effect can be eliminated, depending on context strength. ${ }^{2}$ However, the fundamental argument of whether the language system is modular or interactive remains despite decades of language research. ${ }^{3}$

Perhaps a more productive approach is to identify the factors that will influence the resolution of lexical ambiguity. In addition to the factors of meaning frequency, type of context, and strength of context, Vu et al. (1998) have found that the lexical entries in a simple subject- 
verb-object sentence can provide unique sources of constraint on ambiguity resolution. For example,

2a. The man located the bat.

2b. The slugger located the bat.

2c. The biologist located the bat.

By manipulating the specificity of the subject noun, $\mathrm{Vu}$ et al. (1998) demonstrated that strong biasing contexts immediately activated the contextually appropriate meaning of an ambiguous word (i.e., wood and flying for Sentences $2 b$ and $2 c$, respectively), whereas ambiguous contexts activated both meanings (Sentence 2a). Intralexical priming was excluded as a possible explanation for the data, on the basis of the results of four control experiments (see Vu et al., 1998, as well as the General Discussion section below). Instead, the data were interpreted within a constraint-based feature framework in which incremental processing of words produces an evolving featural representation that, in turn, constrains activation of contextually relevant features of the ambiguous word. Since the stimulus set differed only with respect to the subject noun, Vu et al. (1998) proposed that the resolution process was achieved because of situationbased knowledge associated with nouns. Because a specific subject noun represents a human capable of any activity, "The slugger located the bat" is literally an ambiguous sentence. We theorized that the subject noun invoked a situational representation wherein the specific referent of the subject noun was established in relation to the expected activities of that referring agent (e.g., a slugger searching for his baseball bat). However, no empirical evidence was provided to substantiate claims that situation-based knowledge affected ambiguity resolution.

The primary goal of the present research was to demonstrate that readers possess situation-based knowledge for the entities that participate as agents in contextually defined situations and that these agents, therefore, can be used to evoke a domain of reference that will include only the contextually appropriate meaning of an ambiguous word. A second goal was to show that situationevoking stimuli can be used at different grammatical positions in a linguistic stream to trigger situation-based knowledge, to enable the resolution of lexical ambiguity. Sanford and Garrod (1981) have proposed that a discourse situation can be invoked early or late, depending on the specificity of the words making up a text, stating that "we use a linguistic input to call up representations of situations or events from long-term memory as soon as we have enough information to do so" (p. 115). Following Sanford and Garrod, we suggest that situationbased knowledge can be tapped early or late, as determined by the location of a specific situation-evoking stimulus in a linguistic stream. We evaluated situationevoking stimuli at two grammatical positions (head subject noun and direct object) to determine each position's relative effectiveness in triggering situation-based knowl- edge. To avoid a sentence complexity or sentence length confound, the same syntactic structure (i.e., NP + V + $\mathrm{DO}+\mathrm{PP})$ was used in both experiments. Recent research by McRae et al. (2001) suggests that our evaluation should be successful, since they demonstrated that event structures can be accessed via agents, patients, instruments, and even locations. Lancaster and Barsalou (1997) also demonstrated that event representations are organized in such a way that they can be retrieved by different elements participating in the event and that the use of one source of information (agent) to tap into the event structure led to the retrieval of other sources of information (patient).

\section{EXPERIMENT 1}

In Experiment 1, we examined situation-evoking stimuli at an early NP position, by selecting specific agents that we used in the on-line lexical ambiguity resolution experiment to drive readers' expectations toward specific situations or classes of situations. These situationevoking agents were derived empirically by asking participants in a norming task to rate nouns according to their fit as agents that would lead to these specific situations (see the Method section for full details). For example, for the frame ". . . knew the name of the star," defined in its dominant meaning of celestial body, readers established "the astronomer" as an agent likely to be part of the situation and "the counterfeiter" as an unlikely agent. Since an astronomer was likely to participate in the contextual situation whereas a counterfeiter was not, we assumed that the readers had knowledge about the entities, actions, and objects that participated in general situations. If elements of a situation are organized together, the processing of one element should trigger the situation and enable access to the other, related elements.

Our assumption was tested with an off-line completion task and an on-line naming experiment. If readers' situation-based knowledge for "... knew the name of the star" includes an astronomer as a highly likely participant in the event, then reciprocally, when "The astronomer knew the name of the ..." is processed, similar situation-type representations should be triggered that will enable access to the celestial meaning of star. Consequently, the domain of reference that is established will constrain the readers to generate completions that are generally clustered with situational activities associated with an astronomer. In the naming experiment, if situation-based knowledge about astronomer-type activities are activated, a domain of reference will be provided that will include the semantic attributes of the contextually appropriate meaning of the terminal ambiguous word "star", and thus the probe word representing the celestial meaning should be primed. In contrast, processing of "The counterfeiter knew the name of the ..." will activate a domain of reference that will not include the semantic attributes of either meaning of the ambiguous 
word. For sentence contexts that are headed by agents that are unlikely to participate in the described situations, the incompatibility between the situation-based knowledge evoked by an agent and the meanings activated by the sentence-final ambiguity will lead the language processor to settle on the most frequent meaning of the ambiguous word because, pragmatically, this is the meaning that is most likely intended. Note that for agents that are not likely to participate in the defined situations, the dominant and subordinate situational bias distinction is a nominal one. The situations triggered by sentence frames headed by these agents are so unrelated to the upcoming ambiguity that the bias distinction is nonfunctional and the pattern of priming is predicted to be the same for nominally "dominant" and "subordinate" situational primes (i.e., dominated by meaning frequency).

\section{Method}

Participants. Forty-eight undergraduate volunteers from introductory psychology courses from the University of Kansas participated in the naming experiment for class credit. Another 164 participants from Saint Mary's College and the University of Kansas provided normative data. All the participants were native English speakers with normal or corrected-to-normal visual acuity.

Stimuli. Eighty ambiguous sentence frames were constructed with the structure $\mathrm{NP}+\mathrm{V}+\mathrm{DO}+\mathrm{PP}$, using a transitive verb, a theme/patient in direct object position, and a prepositional phrase terminating with an ambiguous word (e.g., “____ knew the name of the star"). The ambiguous nature of these frames was established by prior research using both off-line and priming measures (Vu, Kellas, \& Metcalf, 2000). The ambiguous words had distinct noun/noun meanings and were selected from the association norms of Nelson, McEvoy, Walling, and Wheeler (1980) and Twilley, Dixon, Taylor, and Clark (1994). The range of meaning frequency for the ambiguous word corpus was .52-.98, with a mean of .73 for the dominant sense. The procedure for defining polarity was adopted from Rayner and Frazier (1989), in which probability values were based only on the two alternatives used in the study. Responses other than the two alternatives were eliminated, so that probability computation summed to 1.00 in all cases.

Stimulus pretesting. There were two aspects of the experimental stimuli that required normative data, due to the following assumptions. The first is that a sentence frame such as ". . . knew the name of the star," defined in its dominant sense of celestial body, will reference a situation that will include an astronomer as a highly likely participating agent. When the frame is defined in its subordinate sense, readers will establish a director as a highly likely participating agent. Second, if situation-based knowledge includes clusters of information about entities, actions, and objects that participate in certain situations, then, reciprocally, the processing of the agent plus the predicate fragment ("The astronomer knew the name of the ...") should activate similar situation-type representations that will impose constraints on what is expected to fulf ill the thematic role of the object of the prepositional phrase. ${ }^{4}$

Situation-evoking agents. Situation-evoking agents were established via a norming method that provided the situation and asked the participants to rate the degree to which potential agents were expected to participate in the situation. However, we used the results of this norming study to provide an agent in a sentence-initial position and then investigated the influence of this agent on computations that occurred later in a sentence that duplicated the situation used in the norming study. Because our focus is the on-line data, we will use the term situation-evoking agent in lieu of expected and unexpected agents for the remainder of the article (after the Method section). ${ }^{5}$
McRae, Ferretti, and Amyote's (1997) methodology for determining agent typicality for verb concepts was adopted, in which the participants established entities that were highly expected to participate as agents in certain situations. For each sentence frame, five potential agents were provided to complete the frame in its dominant-situation bias, and another five agents were provided for the subordinatesituation bias. All agents were initially selected on the basis of agreement among three judges. The agents were selected to include at least one agent that was highly expected to participate in the defined situation and one agent that would be an unexpected participant. The other three agents were selected so as to vary in their degree of expectedness. Two lists were constructed, with 80 ambiguous frames per list (40 in the dominant and 40 in the subordinate reading, with no repetition of ambiguous frames) and five agent fillers per ambiguous frame. For each ambiguous sentence frame, the participants were provided with (1) an intended definition of the terminal ambiguous word and (2) the five potential agents. The participants were asked to read and understand the definition and sentence frame and then to rate each of the five listed agents on the degree to which each agent would be expected to participate in the situation described by the sentence frame (see Example 1).

\section{Example 1}

star: any of the celestial bodies that can be seen at night from Earth.

The knew the name of the star.

\begin{tabular}{|c|c|c|c|c|c|c|c|}
\hline & $\begin{array}{r}\text { ver } \\
\text { unexp }\end{array}$ & & & & & & $\begin{array}{l}\text { very } \\
\text { pected }\end{array}$ \\
\hline counterfeiter & 1 & 2 & 3 & 4 & 5 & 6 & 7 \\
\hline theoretician & 1 & 2 & 3 & 4 & 5 & 6 & 7 \\
\hline navigator & 1 & 2 & 3 & 4 & 5 & 6 & 7 \\
\hline astronomer & 1 & 2 & 3 & 4 & 5 & 6 & 7 \\
\hline geographer & 1 & 2 & 3 & 4 & 5 & 6 & 7 \\
\hline
\end{tabular}

star: a performer widely acknowledged as outstanding.

The knew the name of the star.

\begin{tabular}{lccccccc} 
& $\begin{array}{c}\text { very } \\
\text { unexpected }\end{array}$ & & & & \multicolumn{3}{c}{$\begin{array}{c}\text { very } \\
\text { expected }\end{array}$} \\
cheerleader & 1 & 2 & 3 & 4 & 5 & 6 & 7 \\
confectioner & 1 & 2 & 3 & 4 & 5 & 6 & 7 \\
director & 1 & 2 & 3 & 4 & 5 & 6 & 7 \\
paramedic & 1 & 2 & 3 & 4 & 5 & 6 & 7 \\
photographer & 1 & 2 & 3 & 4 & 5 & 6 & 7 \\
\hline
\end{tabular}

The two lists were reversed to avoid potential order effects, and the four subsets were given to four groups of 12 participants to rate. On the basis of the participants' ratings of agent expectancy, for each unique ambiguous word, four agents were selected from the highest and lowest scores on the expectancy scale (very expected, dominant $M=6.42, S D=0.56$, subordinate $M=6.38, S D=0.50$; unexpected, dominant $M=1.72, S D=0.40$, subordinate $M=$ $1.71, S D=0.50)$.

Referen ce domain and noun filler sentence completion. Since research has demonstrated that entities, actions, and objects are clustered around event structures (e.g., Lancaster \& Barsalou, 1997; McRae et al., 2001), we assumed that processing a specific agent that was established as a highly likely participant of a contextually defined situation would evoke a similar situational representation that would allow access to other situation-based information. The activated situation provides a domain of reference for the interpretation of subsequent information (see Sanford \& Garrod, 1981), and as long as the new information is compatible with and does not conflict with the current information, the situational representation will be updated accordingly, without need for the activation of a new situation (see Zwaan \& Radvansky, 1998). Thus, the situational representation that is evoked when "the astronomer" is encountered will be maintained with the processing of ". . . knew 
the name of the ..." The question arises as to what extent the specific agent, along with the predicate phrase leading up to the object of the preposition, will evoke a similar situational representation with a domain of reference that will contain the targeted lexical ambiguity and/or the intended meaning of the ambiguous word. Following Taraban and McClelland's (1988) procedure for pretesting participants' expectations for prepositional phrase noun fillers, we had the participants perform a modified Cloze task. Importantly, however, rather than have the participants complete the sentence fragment with the first word that came to mind, we gave explicit instructions for generating constrained Cloze completions. The instructions included a definition of a situation and a discussion of how situations often include typical characters, objects, place, time, and so forth and the relationships among the entities involved. The participants were asked to read each sentence fragment without the sentence-final ambiguity, to consider carefully the typical situation(s) in which the specific agent likely would be found, and to use that information to determine the noun filler that would be most likely to terminate the sentence frame.

We predicted that sentence frames headed by specific agents that were established as highly likely agents in the norming study (e.g., "The astronomer knew the name of the ...") would lead the participants to generate sentence completions that would be part of a restricted domain of reference that included either the actual ambiguous words and the probe words used or semantically related referents. For sentence frames headed by specific agents that were established as unlikely agents (e.g., "The counterfeiter knew the name of the ..."), situational representations would be evoked that would lead the participants to generate completions that would be typically clustered with these agents (e.g., bank, criminal, currency, etc.) but would not include the critical ambiguous words, probe words, or words semantically related to the ambiguous words in the domain of reference.

The 320 experimental sentences were separated into two sets of 160 , with pairs of dominant sentences alternating with pairs of subordinate sentences for each set and with the sentence-final ambiguity removed. Two groups of 18 participants were each given a packet of 160 sentence frames to perform the noun filler completion task. For sentence frames headed by agents that were likely to participate in the originally defined situations, the completion data showed that $69.7 \%$ of the generated responses were related to the intended domain of reference (of that, $11.6 \%$ of the completions were the actual ambiguous and probe words used), whereas $30.3 \%$ were unrelated. In contrast, for agents that were unlikely to participate in the original situations, only $3.5 \%$ of the data were related to the targeted reference domain $(0.2 \%$ were the ambiguous and probe words used), whereas $96.5 \%$ of the responses were unrelated. Overall, the noun filler completions showed that agents that were likely to participate in specifically defined situations that included one meaning of an ambiguous word can, in turn, be used to generate similar domains of reference that include the intended meaning of the ambiguous words.

Naming task. Although the norming task established expected agents, in the present naming experiment, it is the sentence-initial agent that drives expectations. Because expected agent might mislead the reader and because our primary focus was the on-line experiment, we adopted the more neutral term agent likelihood to describe the agent-independent variable. Each of the 80 ambiguous sentence frames was paired with a likely dominant biased agent, a likely subordinate biased agent, an unlikely "dominant" agent, and an unlikely "subordinate" agent, for a total of 320 experimental sentence primes. For each ambiguous word, two lexical associates were selected as probe words, one representing a dominant and the other a subordinate meaning of an ambiguous word. Unrelated conditions were created by re-pairing related primes and probes. Thus, the experiment was a $2 \times 2 \times 2 \times 2$ within-subjects design, with factors of agent likelihood (likely or unlikely) $\times$ situation type (dominant or subordinate) $\times$ probe dominance (dominant or subordinate) $\times$ probe relatedness (related or unrelated). In order for each prime and probe to appear only once per participant, with each participant exposed to all the conditions, 16 stimulus lists were constructed. Each list consisted of an equal number of 16 possible prime-probe conditions, using five ambiguous words per condition, resulting in a total of 80 experimental trials per list. In addition, 36 novel sentences with unique ambiguous words were constructed for use as practice trials. These practice trials were representative of the experimental conditions and were identical for all the participants. Table 1 contains an example of the stimuli used for each of the conditions.

The probes were equated across conditions on number of letters (dominant, $M=4.93, S D=1.42$; subordinate, $M=5.11, S D=$ 1.64), syllables (dominant, $M=1.38, S D=0.62$; subordinate, $M=$ $1.49, S D=0.70$ ), bigram frequency (dominant, $M=5,271.0, S D=$ 3,748.5; subordinate, $M=5,228.0, S D=3,975.8$; from Solso \& Juel, 1980), and frequency of occurrence in the English language (dominant, $M=107.9, S D=163.3$; subordinate, $M=110.7, S D=$ 261.4; from Francis \& Kučera, 1982). There was no reliable difference found for any variable (all $p s>.20$ ).

Apparatus. The stimuli were presented on an IBM-compatible 486 personal computer with an NEC Multisync-Plus color monitor. A Shure microphone (Model 515SB), attached to a Grason-Stadler E7300A-1 voice-operated relay (VOR), was interfaced with the computer to signal verbal responses. Stimulus presentation was synchronized to the refresh rate of the monitor, and response times were measured to the nearest millisecond from the onset of the probe to the triggering of the VOR by a vocal response.

Procedure. All the participants were tested individually in a dimly lit room and were seated approximately $60 \mathrm{~cm}$ from the computer monitor, so that probes subtended an average visual angle of

Table 1

Examples of Prime and Probe Stimuli in Experiment 1

\begin{tabular}{|c|c|c|c|}
\hline \multirow{2}{*}{ Situation Primes } & & \multicolumn{2}{|c|}{ Probes } \\
\hline & & Related & Unrelated \\
\hline \multicolumn{4}{|l|}{ Dominant } \\
\hline Likely: & The astronomer knew the name of the star. & sky & church \\
\hline Unlikely: & The counterfeiter knew the name of the star. & & \\
\hline \multicolumn{4}{|l|}{ Subordinate } \\
\hline Likely: & The director knew the name of the star. & movie & head \\
\hline Unlikely: & The confectioner knew the name of the star. & & \\
\hline \multicolumn{4}{|l|}{ Dominant } \\
\hline Likely: & The rabbi discussed the damage to the temple. & church & sky \\
\hline Unlikely: & The astronomer discussed the damage to the temple. & & \\
\hline \multicolumn{4}{|l|}{ Subordinate } \\
\hline Likely: & The physician discussed the damage to the temple. & head & movie \\
\hline Unlikely: & The vegetarian discussed the damage to the temple. & & \\
\hline
\end{tabular}


about $1.6^{\circ}$ horizontally and $0.5^{\circ}$ vertically. The participants completed the experiment in about $20 \mathrm{~min}$.

The stimuli were presented in a cumulative manner (see Just, Carpenter, \& Woolley, 1982) in which the words making up a sentence context were presented one at a time across the computer screen, with the words remaining visible until the final word was displayed. This unfolding procedure has been employed successfully in previous studies in which on-line language processing was examined (e.g., Dell, McKoon, \& Ratcliff, 1983; Greene, McKoon, \& Ratcliff, 1992; Paul et al., 1992; Vu, Kellas, et al., 2000; Vu et al., 1998). For the present research, a $235-\mathrm{msec}$ presentation rate was employed that was comparable to the presentation rates used in other on-line research on reading comprehension, from $250 \mathrm{msec}$ (e.g., Dell et al., 1983; Duffy, Henderson, \& Morris, 1989; Greene et al., 1992) to $500 \mathrm{msec}$ (e.g., Stanovich \& West, 1983). This presentation rate is also within the range for first-pass eye fixations $(\sim 200-250 \mathrm{msec})$ found for critical words in reading comprehension research in which eye-tracking methodology has been used (e.g., Duffy et al., 1988).

For each trial, a series of word-length lines, each separated by a single space, was presented for $1 \mathrm{sec}$. The lines served as a warning signal and as location and length cues for the words constituting the upcoming sentence. Each sentence was presented beginning at the left side and vertical center of the computer screen. Sentence primes were displayed in lowercase letters (with the exception of the first letter of the first word, which was capitalized). The participants were instructed to read the presented sentences silently and to read for comprehension. Immediately following the sentencefinal ambiguous word (0-msec interstimulus interval [ISI]), the sentence was removed, and a probe word was displayed six character spaces to the right of where the sentence had previously ended. Probe words were presented in uppercase letters in order to be visually distinguishable from the sentences. The participants were instructed to name the probe word aloud as quickly and as accurately as possible. To safeguard against the strategy of not reading for comprehension, the participants were asked to answer $w h$-comprehension questions on a random $50 \%$ of the trials.

Naming responses were monitored for accuracy. Response errors included incomplete responses, mispronunciatio ns, extraneous noises, and artificial delays resulting from responses that initially failed to trigger the VOR. All the trials were separated by a 2,500$\mathrm{msec}$ intertrial interval, and responses to the first 36 naming trials constituted practice and were not examined.

\section{Results}

Naming latencies that were above $1,400 \mathrm{msec}$ and below $300 \mathrm{msec}$ were removed $(0.40 \%)$, and response errors constituted another $2.24 \%$ of the data. Comprehension accuracy was $85 \%$.
Mean correct latencies were submitted to an agent likelihood (likely or unlikely) $\times$ situation type (dominant or subordinate) $\times$ probe dominance (dominant or subordinate) $\times$ probe relatedness (related or unrelated) analysis of variance (ANOVA) for repeated measures, with both participants $\left(F_{1}\right)$ and items $\left(F_{2}\right)$ as random factors. All effects reported were significant at $p<.05$, unless otherwise indicated. Mean latencies, percentage of errors, and the magnitude of priming are shown in Table 2.

Table 2 shows that naming latencies for contextually appropriate probe words following highly likely dominant and subordinate biased situations were facilitated relative to unrelated conditions, whereas latencies for contextually inappropriate probes were not. In contrast, naming latencies for dominant probe words were facilitated following nominally "dominant" and "subordinate" biased situations, as compared with unrelated conditions. Subordinate probe words were not primed by either the "dominant" or the "subordinate" biased situations. This description is supported by the following statistical outcomes.

On the basis of a priori predictions, the three-way situation type $\times$ probe dominance $\times$ probe relatedness interaction was examined for each level of agent likelihood separately. For very likely agents, analyses revealed reliable effects of probe relatedness and probe dominance, which was qualified by a situation type $\times$ probe dominance $\times$ probe relatedness interaction $\left[F_{1}(1,47)=\right.$ $\left.4.85, M S_{\mathrm{e}}=2,320.6 ; F_{2}(1,79)=4.55, M S_{\mathrm{e}}=3,953.5\right]$. No other main effects or interactions were significant. The three-way interaction was examined further by evaluating probe dominance $X$ probe relatedness for each level of situation type separately. For the dominant biased situation, there was an effect of probe relatedness that was qualified by the interaction of probe dominance and probe relatedness $\left[F_{1}(1,47)=5.27, M S_{\mathrm{e}}=1,766.8\right.$; $\left.F_{2}(1,79)=4.14, M S_{\mathrm{e}}=4,166.6\right]$. As is indicated in Table 2, contextually appropriate dominant probes were facilitated following dominantly biased situations, relative to unrelated probes $[t(47)=3.92, p<.01]$. On the other hand, contextually inappropriate subordinate probes were not facilitated relative to unrelated probes $[t(47)=0.67, p>.20]$. An examination of the subordi-

Table 2

Mean Naming Latencies (in Milliseconds), Percentages of Error, and Magnitude of Priming (Unrelated - Related) for Experiment 1

\begin{tabular}{|c|c|c|c|c|c|c|c|}
\hline \multirow{3}{*}{$\begin{array}{c}\text { Agent } \\
\text { Likelihood }\end{array}$} & \multirow[b]{3}{*}{ Bias } & \multirow{3}{*}{$\begin{array}{c}\text { Probe } \\
\text { Dominance }\end{array}$} & \multicolumn{4}{|c|}{ Probe Relatedness } & \multirow[b]{3}{*}{ Priming } \\
\hline & & & \multicolumn{2}{|c|}{ Related } & \multicolumn{2}{|c|}{ Unrelated } & \\
\hline & & & $M$ & $\overline{\% \mathrm{E}}$ & $M$ & $\overline{\% \mathrm{E}}$ & \\
\hline \multirow[t]{4}{*}{ Likely } & Dom & Dom & 665 & 0.8 & 699 & 2.1 & $34 *$ \\
\hline & & Sub & 688 & 4.2 & 694 & 1.3 & 6 \\
\hline & Sub & Dom & 698 & 3.3 & 708 & 2.5 & 10 \\
\hline & & Sub & 677 & 3.3 & 703 & 2.9 & $26^{*}$ \\
\hline \multirow[t]{4}{*}{ Unlikely } & Dom & Dom & 705 & 1.7 & 732 & 4.6 & $27 *$ \\
\hline & & Sub & 705 & 1.7 & 702 & 2.1 & -3 \\
\hline & Sub & Dom & 701 & 3.3 & 734 & 3.8 & $34 *$ \\
\hline & & Sub & 709 & 2.9 & 722 & 1.7 & 13 \\
\hline
\end{tabular}

Note-Dom, dominant; Sub, subordinate. * Significant, $p<.05$ 
nate biased situations showed an opposite pattern of activation. The results showed that contextually appropriate subordinate probes were facilitated relative to unrelated probes $[t(47)=2.35, p<.05]$. In contrast, responses to dominant probes were not facilitated relative to unrelated probes $[t(47)=0.95, p>.50]$.

Analysis for unlikely agents showed an effect of probe relatedness $\left[F_{1}(1,47)=9.94, M S_{\mathrm{e}}=2,945.1 ; F_{2}(1,79)=\right.$ $\left.8.05, M S_{\mathrm{e}}=4,556.3\right]$ and a probe dominance $\times$ probe relatedness interaction $\left[F_{1}(1,47)=4.72, M S_{\mathrm{e}}=3,268.7\right.$; $\left.F_{2}(1,79)=9.77, M S_{\mathrm{e}}=3,918.6\right]$, but the situation type $\times$ probe dominance $\times$ probe relatedness interaction was not significant $\left[F_{1}(1,47)=0.33, M S_{\mathrm{e}}=1,815.0 ; F_{2}(1,79)=\right.$ $\left.0.01, M S_{\mathrm{e}}=3,455.4\right]$. Planned comparisons showed that for dominant biased situations, dominant probes were facilitated, as compared with unrelated probes $[t(47)=2.10$, $p<.05]$, but subordinate probes were not $[t(47)=0.50$, $p>.50]$. For subordinate biased situations, the difference between dominant probes and unrelated probes was significant $[t(47)=3.54, p<.01]$, but not that between subordinate probes and unrelated probes $[t(47)=1.37$, $p>.15] .6$

\section{Discussion}

The norming study confirmed that readers possess knowledge regarding the likelihood of various agents for specific situations. Experiment 1, the on-line naming study, showed that these situation-evoking agents, which were placed in the sentence-initial position, triggered situational representations that included the targeted lexical ambiguity, as well as words related to the intended meaning of the lexical ambiguity. Agents that were likely to participate in their respective dominant or subordinate biased situations activated appropriate domains of reference for the resolution of lexical ambiguity. In contrast, unlikely agents tapped into situations that were not immediately compatible domains of reference for interpreting the final ambiguity. For example, when "The counterfeiter knew the name of the ..." was processed, situation-based knowledge led the reader to expect concepts related to a counterfeiter to fill the object of the prepositional phrase. ${ }^{7}$ When star was processed, neither of its meanings satisfied the situational-level constraint. The dominant meaning ramped up faster, due to meaning frequency, and the language processor settled on this probabilistically intended meaning.

\section{EXPERIMENT 2}

Sanford and Garrod (1981) theorized that a situation is evoked as early as possible, but only when there is sufficiently specific information to do so. When situations are evoked, it is important to use salient cues in order to facilitate retrieval of relevant information from long-term memory. Linguistic expressions can activate situationspecific knowledge, and the type of situation that is activated depends on the specificity of the roles and actions involved. Thematic roles instantiated with general terms will activate only poorly defined situations or no situa- tion at all, and the reader must await further information before establishing an appropriate domain of reference. However, more specific thematic role fillers will activate a situation rich with information for interpreting subsequent linguistic expressions. This suggests that a situation can be evoked further into a linguistic stream than at the (well-accepted) verb position or the agent position.

In Experiment 2, we used situation-evoking stimuli at a later direct object position to examine lexical ambiguity resolution. The same syntactic structure as that in Experiment $1(\mathrm{NP}+\mathrm{V}+\mathrm{DO}+\mathrm{PP})$ was used, and all the words leading up to the direct object were general terms. The specificity of the theme/patient role filler was manipulated, where the linguistic expressions were either specific or general with respect to a given contextual situation. The predictions were that contexts with specific situation-evoking stimuli (e.g., "She knew the galaxy of the star") would produce a strongly biased sentence context that would activate a restricted domain of reference that included only attributes of the intended meaning of an ambiguous word; however, contexts with nonspecific role fillers (e.g., "She knew the name of the star") would produce an ambiguous situation with a broad domain of reference that was supportive of both meanings of an ambiguous word. Thus, naming latencies to probe words were expected to show that both meanings of an ambiguous word would be primed following ambiguous contexts. In contrast, only the contextually appropriate meaning would be primed following strongly biased contexts (dominant or subordinate).

\section{Method}

Participants. Forty-eight undergraduate volunteers from introductory psychology courses participated in the naming experiment for class credit. An additional 108 participants provided strengthof-context ratings. All the participants were native English speakers with normal or corrected-to-normal visual acuity.

Stimuli. One hundred fifty-eight ambiguous words with distinct noun/noun meanings were selected from the association norms of Nelson et al. (1980) and Twilley et al. (1994). Three sentence contexts (strong dominant biased, strong subordinate biased, and ambiguous) were constructed for each homonym, resulting in a total of 474 sentences. The structure of all three context types was identical, with an ambiguous word terminating the prepositional phrase. Across the three context types, only the noun phrase fulfilling the theme/patient role of the direct object was free to vary, whereas all the other constituents were identical. Thus, the bias of the sentence contexts was determined only by the manipulation of the theme/patient role fillers. Two lexical associates were selected as probe words for each homonym, one representing the dominant and the other a subordinate meaning of the ambiguous word.

The particular bias of the experimental sentences was initially agreed upon by three judges. The sentences were subsequently divided into three sets of 158 each, with no repetition of an ambiguous word in any set and each set containing an approximately equal number of dominant, subordinate, and ambiguous sentences. For each set, presentation of the 158 sentences was reversed for one half of the participants to avoid potential order effects. All six subsets were presented to six groups of 18 participants each for normative bias ratings. The participants rated the degree to which each sentence was biased toward the associatively related probe words on a 9-point scale (where 1 represented a strong bias toward the dominant meaning and 9 represented a strong bias toward the subordinate 
meaning). Strict criteria were used in selecting the experimental stimuli from the participants' ratings. For each sentence triad to be selected, strongly biased dominant sentences had to be rated between 1.00 and $3.00(M=1.72, S D=0.48)$, ambiguous sentences between 3.10 and $6.90(M=4.84, S D=0.32)$, and strongly biased subordinate sentences between 7.00 and $9.00(M=8.13, S D=$ $0.51)$. The nonoverlapping distributions ensured clear boundaries between prime conditions. On the basis of the above criteria, 84 sentence triads ( 252 sentences) were selected for study.

Each sentence prime (dominant, subordinate, or ambiguous) was paired with two related probe words (dominant or subordinate). The ambiguous words and the probe words were identical to those in Experiment 1. The stimulus characteristics were identical to those in Experiment 1, including meaning frequency. Unrelated conditions were created by re-pairing related primes and probes. In order for each prime and probe to appear only once per participant, with each participant exposed to all conditions, 12 stimulus lists were constructed. Each list consisted of an equal number of 12 possible prime-probe conditions, using seven homonyms per condition, resulting in a total of 84 experimental trials per list. In addition, 36 sentences with unique homonyms were taken from the remaining 158 rated sentences, for use as practice trials. The 36 practice trials were representative of the experimental conditions and were identical for all the participants. Table 3 contains an example of the stimuli used for each of the conditions.

Apparatus and Procedure. The apparatus and procedure were the same as those in Experiment 1.

\section{Results}

Two participants were replaced due to a high rate of comprehension errors $(>40 \%)$, and 2 participants were replaced due to excessive naming errors $(>10 \%)$. Response errors constituted only $5.3 \%$ of the data, and comprehension accuracy was $90.9 \%$.

Mean correct naming latencies were submitted to a prime type (ambiguous, dominant bias, or subordinate bias) $\times$ probe dominance (dominant or subordinate) $\times$ probe relatedness (related or unrelated) ANOVA for repeated measures, with both participants $\left(F_{1}\right)$ and items $\left(F_{2}\right)$ as random factors. All effects reported were significant at $p<.05$, unless otherwise indicated. Mean latencies, percentages of errors, and the magnitude of priming are shown in Table 4.

Table 4 shows that naming latencies for contextually appropriate probe words following both strongly biased dominant and subordinate primes were facilitated relative to unrelated conditions, whereas latencies for contextually inappropriate probes were not. In contrast, naming latencies for both dominant and subordinate probe words were facilitated following ambiguous primes, as com-

Table 3

Examples of Prime and Probe Stimuli in Experiment 2

Probes

Situation Primes $\overline{\text { Related Unrelated }}$

Amb: She knew the name of the star.

Dom: She knew the galaxy of the star.

Sub: She knew the wife of the star. sky church

Amb: He discussed the damage to the temple.

Dom: He discussed the pilgrimage to the temple. church sky

Sub: He discussed the injury to the temple. head movie

Note-Amb, ambiguous; Dom, dominant; Sub, subordinate.
Table 4

Mean Naming Latencies (in Milliseconds), Percentages of Errors, and Magnitude of Priming (Unrelated - Related) for Experiment 2

\begin{tabular}{|c|c|c|c|c|c|c|}
\hline \multirow{3}{*}{$\begin{array}{l}\text { Prime } \\
\text { Type }\end{array}$} & \multirow{3}{*}{$\begin{array}{l}\text { Probe } \\
\text { Type }\end{array}$} & \multicolumn{4}{|c|}{ Probe Relatedness } & \multirow[b]{3}{*}{ Priming } \\
\hline & & \multicolumn{2}{|c|}{ Related } & \multicolumn{2}{|c|}{ Unrelated } & \\
\hline & & $M$ & $\% \mathrm{E}$ & $M$ & $\% \mathrm{E}$ & \\
\hline \multirow[t]{2}{*}{ Ambiguous } & Dom & 645 & 4.5 & 666 & 4.2 & $21 *$ \\
\hline & Sub & 647 & 3.3 & 664 & 5.1 & $17 *$ \\
\hline \multirow[t]{2}{*}{ Dominant } & Dom & 651 & 7.4 & 674 & 6.8 & $23^{*}$ \\
\hline & Sub & 658 & 5.7 & 657 & 6.3 & -1 \\
\hline \multirow[t]{2}{*}{ Subordinate } & Dom & 670 & 3.9 & 672 & 7.1 & 2 \\
\hline & Sub & 645 & 3.6 & 666 & 6.3 & $21 *$ \\
\hline
\end{tabular}

Note-Dom, dominant; Sub, subordinate. *Significant, $p<.05$.

pared with unrelated conditions. This description is supported by the following statistical outcomes.

Analyses revealed reliable effects of probe relatedness, probe dominance, and prime type. However, these effects were qualified by the prime type $\times$ probe dominance $\times$ probe relatedness interaction $\left[F_{1}(2,94)=3.35\right.$, $\left.M S_{\mathrm{e}}=1,356.74 ; F_{2}(2,166)=3.20, M S_{\mathrm{e}}=3,747.13\right]$. No other interactions were significant. The three-way interaction was examined further by evaluating probe dominance $\times$ probe relatedness for each prime type separately. For the dominant prime condition, there was a significant effect of probe relatedness $\left[F_{1}(1,47)=5.69\right.$, $M S_{\mathrm{e}}=973.02$; but $F_{2}(1,83)=2.04, M S_{\mathrm{e}}=4,632.86$, $p=.16]$, qualified by a probe dominance $\times$ probe relatedness interaction $\left[F_{1}(1,47)=5.01, M S_{\mathrm{e}}=1,272.62\right.$; but $\left.F_{2}(1,83)=2.27, M S_{\mathrm{e}}=4,166.68, p=.14\right]$. As is indicated in Table 4, dominant probes were facilitated following strongly biased dominant primes, relative to unrelated probes $[t(47)=2.89, p=.006]$. On the other hand, subordinate probes were not facilitated relative to unrelated probes $[t(47)=0.26, p=.800]$.

An examination of the subordinate prime condition showed an opposite pattern of activation. There were effects of probe relatedness $\left[F_{1}(1,47)=15.33, M S_{\mathrm{e}}=\right.$ $\left.995.59 ; F_{2}(1,83)=6.85, M S_{\mathrm{e}}=4,413.69\right]$ and probe dominance $\left[F_{1}(1,47)=6.32, M S_{\mathrm{e}}=1,623.45 ; F_{2}(1,83)=\right.$ $\left.3.64, M S_{\mathrm{e}}=5,604.12, p=.06\right]$, which were qualified by an interaction of probe dominance and probe relatedness $\left[F_{1}(1,47)=5.60, M S_{\mathrm{e}}=934.31 ; F_{2}(1,83)=3.34, M S_{\mathrm{e}}=\right.$ $4,418.48, p=.07]$. Here, subordinate probes were facilitated relative to unrelated probes $[t(47)=3.99, p<.001]$. Responses to dominant probes, however, were not facilitated $[t(47)=1.35, p=.185]$.

Finally, for the ambiguous prime condition, the only reliable effect was that of probe relatedness $\left[F_{1}(1,47)=\right.$ $\left.14.20, M S_{\mathrm{e}}=1,659.91 ; F_{2}(1,83)=7.65, M S_{\mathrm{e}}=4,993.25\right]$. This outcome indicated that responses to probes related to both meanings of an ambiguous word were facilitated, relative to unrelated probes [dominant, $t(47)=3.63, p=$ .001 ; subordinate, $t(47)=2.39, p=.021]$.

\section{Discussion}

The results of Experiment 2 confirmed that situationevoking stimuli occurring later at the direct object posi- 
tion can evoke a situational representation that provides an appropriate domain of reference for the resolution of a lexically ambiguous word. Specific role fillers produced strongly biased dominant and subordinate contexts that placed constraints on what meaning of an ambiguous word could fulfill the thematic role of the object of the prepositional phrase. For the sentence, "She knew the galaxy of the star," when the terminal ambiguous word was processed, its dominant meaning of a celestial body satisfied the situational constraints imposed by the prior context, and the ambiguity was resolved. In contrast, sentence contexts with more general role fillers were rated as being ambiguous, and no situational constraints were placed on the ambiguous word. When the sentence "She knew the name of the star" was processed, no specific situation was activated, and thus, no constraining frame of reference was provided to resolve the lexical ambiguity.

The specific theme/patient role fillers of Experiment 2 and the specific agent role fillers that were likely to participate in specifically defined situations in Experiment 1 demonstrated a similar pattern of priming - activation of only the contextually appropriate meaning of an ambiguous word. However, the pattern of priming for nonspecific role fillers (Experiment 2) differed from that of specific agents that were not likely to participate in a specifically defined situation (Experiment 1). In Experiment 1, priming of probe words was dominated by meaning frequency, but in Experiment 2, a pattern of multiple activation ordered by meaning frequency emerged. The results are intriguing, since the two experiments share the same ambiguous sentence frames (e.g., ". . . knew the name of star").

We propose that situation-based knowledge and semantic specificity of the role fillers may play a role in explaining the different patterns of activation. In a sentence such as "She knew the name of the star," there is no commitment made to a specific situation when "she" is processed. The semantic representation is general, $<$ female $>$ $<$ animate $>$, and incremental interpretation of the subsequent constituents adds only more general features to the evolving representation. No expectations are established, and without any processing constraints on upcoming words, when the ambiguous word star is processed, both of its alternative meanings will map equally well with the general text representation (a female knowing the name of a celestial body or a performer). Thus, the sentence is rated as being ambiguous and primes both meanings of the ambiguous word. For the sentence "The counterfeiter knew the name of the star," although it is literally ambiguous, a commitment is a made to a specific situation when "the counterfeiter" is processed. The initial representation would include, at least, $<$ person $>$ $<$ criminal $><$ money $><$ fraud $><$ etc. $>$. This specific header should be sufficient to tap into situation-based knowledge that readers have about counterfeiters. Thus, counterfeiter-type activities are expected that will provide processing constraints on what a counterfeiter should know, such as banks, money, other criminals, and so forth (see note 7). When the fragment "The counterfeiter knew the name of the ..." is processed, it is highly likely that concepts associated with counterfeiting are expected to fill the thematic role of the object of the prepositional phrase. These concepts are preactivated by the prior context. However, when the ambiguous word "star" is processed, its alternative meanings (sky and movie) are so unexpected that the language processor will quickly commit to the dominant meaning, since that meaning is most probabilistically and pragmatically intended.

\section{GENERAL DISCUSSION}

The results of Experiments 1 and 2 demonstrated that situation-evoking stimuli can occur at an early head NP or later in a linguistic stream, at the DO position, and can tap into situational structures to provide a domain of reference for the interpretation of subsequent (ambiguous) linguistic information. In Experiment 1, readers established likely and unlikely participating agents for specifically defined contextual situations that included one meaning of an ambiguous word. Agents that were likely to participate in a defined situation evoked a domain of reference that included semantic attributes that overlapped with the contextually appropriate meaning of the terminal lexical ambiguity. Unlikely agents of a defined situation evoked a domain of reference that did not share semantic attributes with either meaning of the ambiguous word. In these situations, meaning frequency dominated the resolution process. Experiment 2 indicated that the specificity of the filler selected to instantiate the theme/patient role determined whether a sentence was strongly biased and evoked a specific situation to resolve the lexical ambiguity or whether the context was ambiguous and supported both meanings of the ambiguous word.

The results of the present research are compatible with, and extend, our previous work. Vu et al. (1998) employed a systematic componential analysis of linguistic contexts to determine the linguistic sources of constraint on the resolution of lexical ambiguity. With a simple manipulation of the specificity of the verb or the subject noun, we demonstrated that each linguistic component provided a unique source of constraint on lexical processing, via situational representations, and that the degree of constraint that was imposed on ambiguity resolution depended on the specificity of the component. Thus, combining the present research and that of $\mathrm{Vu}$ et al. (1998), we have tracked situation-evoking stimuli across the agent position, the verb position, and the direct object position in simple sentences. We have provided strong corroborating evidence for Sanford and Garrod's (1981) proposals that (1) language comprehension requires the activation of a situation to provide a domain of reference for interpreting subsequent information, (2) this situation can be evoked at any point of a 
linguistic stream, as long as there are specific linguistic expressions, and (3) the reference domain will be updated, refined, and revised with the processing of subsequent information. We interpret our results within the framework of a contextual-feature-sensitive model instantiated as a constraint satisfaction network concatenated incrementally across words making up a sentence context. ${ }^{8}$

\section{Contextual-Feature-Sensitive Model and Incremental Processing}

We start with the assumption that language comprehension occurs incrementally and that semantic memory is represented as a landscape of activated features. Words are represented by clusters of features, and each feature (or set of features) participates in the meaning of many words. The pattern of activation across the network is initially at baseline (or as much as can be with constant external input and internal thoughts) until a linguistic input is encountered. When a word is processed, the landscape of the network will change (become activated) to reflect the input. The area of activation will be broadly dispersed or more focused and narrow, depending on the specificity of the input. As more words are analyzed, the landscape will change dynamically to reflect each additional input. Presumably, the processing of each word activates a pattern of features that is constrained, in cascade, by prior discourse, its grammatical function, and its thematic role, as well as by general world knowledge, and that represents the meaning of that word at that point in time (cf. Kawamoto, 1993; McClelland, St. John, \& Taraban, 1989). These features are simultaneously mapped onto a semantic representation of the discourse. The discourse representation is modified with the mapping of each constituent, in that semantic features that are relevant for the ongoing discourse remain activated, while less salient or inappropriate features are dampened. In addition, situation-based knowledge about entities and events may be incorporated with the context, and this information will impose further constraints on the evolving representation (e.g., Johnson-Laird, 1983; Sanford \& Garrod, 1981). During stimulus analysis of a word form, a pattern of activation representing all the features of a word is initially computed, due to bottomup priority (cf. Marslen-Wilson, 1987). However, the semantic representation that has been computed for the prior context will have preactivated features that may be shared with an upcoming word. When a subsequent word is processed, its semantically related features will partially overlap with the discourse representation, in a convergence of top-down and bottom-up processing, and these features are quickly computed to a recognition threshold. Thus, the spotlight of activation can become increasingly more concentrated on a delimited set of specific features and, consequently, can restrict the domain of reference. But the activation is sensitive to the priority of the most recent linguistic input. That is, although incremental processing may constrain the activation and interpretation of a subsequent word, it is the actual lexical entry that determines the fate of the evolving representation. Context can project the next appropriate word only via activated features, which may or may not be realized by the input. If the lexical entry shares activated features with the situational representation, processing will be expedited; if not, resolution of the meaning of the sentence will be delayed by the increased time for the network to settle. Following a sentence, the messagelevel representation, in combination with content-based expectancies for constituent structure and thematic role assignment (Taraban \& McClelland, 1988), will guide the interpretation of the first content word in the next clause or sentence. The process continues incrementally to develop a running discourse-level representation. In our view, text processing is a matter of a concatenation of individual processing episodes.

In recent years we have proposed a contextual-featuresensitive model of language processing to provide a framework for our research (e.g., Kellas, Paul, Martin, \& Simpson, 1991; Martin et al., 1999; Paul et al., 1992; Vu, Kellas, et al., 2000; Vu et al., 1998). According to this constraint-based architecture, the understanding of the intended meaning of an ambiguous word (or the intended sense of a polysemous word) is sensitive to the context in which it occurs. A context must be strongly biased in order to emphasize the intended meaning; if a context is ambiguous or weakly biased, there is no commitment to a specific semantic interpretation, and multiple meanings (and/or senses) will be available. Incremental processing of words will combine constraints from multiple sources of information (sentential and extrasentential) that will converge on an ambiguity. Depending on the number and the strength of the constraints, a context can be computed that will be neutral (allowing a single frequent meaning to dominate), ambiguous and supportive of multiple meanings, strongly biased and constraining one meaning, or completely predictive so that only one word will satisfy the constraints. 9 Theoretically, when all sources of information and, thus, all constraints converge at a single point in time and semantic space, an upcoming word's meaning is rendered predictable. Thus, multiple activation, activation of multiple meanings ordered by frequency, or selective activation is possible, depending on the constraints imposed on the computational process. It is this dynamic multipleconstraint process that provides the mechanism for ambiguity resolution in Experiments 1 and 2.

A potential alternative explanation for the pattern of priming found in Experiments 1 and 2 is that of intralexical priming from content word(s) in the experimental sentences to the probe words. However, as we have argued elsewhere (e.g., Vu et al., 1998), although word-based priming occurs with lexical associates in isolation, the magnitude and duration of priming found in discourse processing extends beyond that of simple intralexical priming. For example, Foss (1982), Gough, Alford, and Holley-Wilcox (1981), and O'Seaghdha 
(1989) have all demonstrated that priming of two lexical associates diminishes with the intervention of one or more unrelated lexical items. In addition, $\mathrm{Vu}$ et al. (1998) found contextual priming of the probe target "station" following the context "The soldier patrolled the base." An argument was made that the presence of the lexical associates "soldier" and "patrolled" primed "station." In a strong demonstration against word-based priming, Vu et al. (1998) conducted one experiment in which the sentence representation was modified ("The soldier patrolled the streets") and another experiment in which semantic and syntactic coherence was eliminated ("soldier the the patrolled base"). In both experiments, the lexical associates and probes were maintained, but in neither control experiment could intralexical priming explain the results of the formal experiments. Alternatively, it has been suggested that soldier primes base, which, in turn, primes station. However, this associative priming hypothesis is problematic, because base is associatively related to both meanings of the ambiguity (dominant, safe, as in the baseball sense; subordinate, station, as in the military sense). If one assumes that the associative connection is stronger for the dominant sense than for the subordinate sense, the associative priming hypothesis predicts that safe should be more strongly primed than station or that, minimally, both senses should be primed. However, the empirical effect was that only the subordinate sense of the ambiguity was primed in strong subordinately biased contex ts but that multiple meaning frequency ordered activation occurred for the scrambled version. A strong associative priming hypothesis predicts identical outcomes for both normal and scrambled versions, and this did not occur. Even if the associative hypothesis were ensconced within a spreading activation account, there would still be the problem of selective activation. In a semantic network such as that proposed by Collins and Loftus (1975), the concept node representing base, when activated, should spread activation to related concept nodes (safe, station). To account for a selective outcome, it would be necessary, at minimum, to have inhibitory connections between competing nodes. This would require a modification to the model. In the present research, it seems unlikely that the addition of a simple prepositional phrase would substantially alter the outcome. Strictly speaking, however, the present research did not employ scrambled sentences, so we have relied on logical extension. It may well be that an associative priming and/or spreading activation framework can be proposed to account for the present outcome. However, as a general model, there will be some very subtle effects that will have to be accommodated. For example, Ferretti (2000) demonstrated that a verb in its imperfective aspect "was skating" primed a location "arena," whereas a verb in its perfect aspect "had skated" did not prime the location. A spreading activation account of intralexcial priming cannot obviously account for these results. Evidence against word-based priming in linguistic contexts has been provided by nu- merous researchers, using different methodologies, including behavioral measures (e.g., Foss \& Speer, 1991; Hess et al., 1995; Simpson, Peterson, Casteel, \& Burgess, 1989), eye tracking (e.g., Dopkins et al., 1992; Traxler et al., 2000), and ERPs (e.g., Coulson et al., 2000), to name a few (see also McRae et al., 2001, for theoretical discussions against traditional word-based priming as a viable mechanism by which to explain situation-based priming).

Finally, it has been suggested that our results and subsequent interpretation may be compromised because the naming paradigm may not be suitably sensitive for investigating the time course of ambiguity resolution. Although this may or may not be true, we submit that there is currently no single paradigm that will provide an unequivocal examination of the temporal resolution of lexical ambiguity. A preferred methodology that is often cited is that of eye tracking and gaze durations. However, analysis of gaze durations does not reveal what meanings have been activated while a reader fixates on an ambiguous word. It is logical to assume that longer gaze duration on an ambiguous word reflects activation of multiple meanings, yet this is an inference drawn in the absence of direct evidence. Moreover, research by Rayner and Well (1996) and Altarriba, Kroll, Scholl, and Rayner (1996) demonstrated that the pattern of data from eye fixations was corroborated by naming latencies. Martin et al. (1999) used self-paced reading as an analogue to gaze durations, along with a naming task, and provided evidence for context sensitivity in ambiguity resolution. This research was replicated by Binder and Rayner (1998), using gaze durations. There are several issues that separate Binder and Rayner (1998) from Martin et al.; however, in terms of methodology, the replication confirms the validity of the naming task as a sensitive on-line measure of language processing. Unfortunately, there is no current methodology that can isolate the pure, discrete processing stages that are required of strict modularity. Even ERP data suggest that meaning integration can begin before the completion of word recognition (e.g., Van Petten, Coulson, Rubin, Plante, \& Parks, 1999). Thus, at this point, it is impossible to conclusively determine whether the language system is one of immediate interactivity or fast modularity.

\section{Conclusion}

Our past and present research provides strong empirical support for a contextual-feature-sensitive model of language processing. We have found that agent roles, verbs, theme/patient roles, meaning frequency, strength of context, and discourse-level information all can affect the processing and resolution of an ambiguous word. We have even demonstrated that information from a prior processing episode can affect the processing of an ambiguous word in a current episode (Martin et al., 1999; Vu, Kellas, et al., 2000). The present results demonstrate that language comprehension is achieved, in part, by a dynamic interaction between the streaming linguistic input and the activation of referential situations. Situation- 
evoking stimuli are necessary to trigger a situational representation and provide a frame of reference for the interpretation of subsequent text. The situation can be activated at any point in a linguistic stream, so long as the evoking stimulus is specific and is continuously updated from incremental processing of the text. Our componential analysis of linguistic contexts has demonstrated that a single lexical alteration can bias the language processor to favor one, the other, or both meanings of an ambiguous word. In future research, we want to demonstrate that situation-based knowledge alone can set up processing constraints and guide language processing. For example, given the situation "The bullet hit the man in the heart/leg. He immediately ____," participants should expect and respond faster to the verb "died" than "fell" when the bullet strikes the man in the heart. However, processing speed should be reversed for the verbs when the man is struck in the leg. Any processing difference would be based solely on the general world knowledge that participants possessed about the consequence of bullets striking specific body parts.

\section{REFERENCES}

Altarriba, J., Kroll, J. F., Scholl, A., \& Rayner, K. (1996). The influence of lexical and conceptual constraints on reading mixedlanguage sentences: Evidence from eye fixations and naming times. Memory \& Cognition, 24, 477-492.

Altmann, G. T. M., \& Kamide, Y. (1999). Incremental interpretation at verbs: Restricting the domain of subsequent reference. Cognition, 73, 247-264.

Binder, K. S., \& RAYNer, K. (1998). Contextual strength does not modulate the subordinate bias effect: Evidence from eye fixations and self-paced reading. Psychonomic Bulletin \& Review, 5, 271-276.

Binder, K. S., \& RAYNER, K. (1999). Does contextual strength modulate the subordinate bias effect? A reply to Kellas and Vu. Psychonomic Bulletin \& Review, 6, 518-522.

Bower, G. H., Black, J. B., \& Turner, T. J. (1979). Scripts in memory for text. Cognitive Psychology, 11, 177-220.

Carpenter, P. A., Miy ake, A., \& Just, M. A. (1995). Language comprehension: Sentence and discourse processing. Annual Review of Psychology, 46, 91-120.

Collins, A. M., \& Loftus, E. F. (1975). A spreading activation theory of semantic processing. Psychological Review, 82, 407-428.

Coulson, S., Van Petten, C., Federmeier, K., Folstein, J., WeckERLY, J., \& KuTAS, M. (2000, March). Lexical and sentential context effects: An ERP study of the difference between life and death and life in prison. Paper presented to the 13th Annual CUNY Conference, San Diego.

Dell, G. S., McKoon, G., \& Ratcliff, R. (1983). The activation of antecedent information during the processing of anaphoric reference in reading. Journal of Verbal Learning \& Verbal Behavior, 22, 121-132.

Dopkins, S., Morris, R. K., \& RAYner, K. (1992). Lexical ambiguity and eye fixation in reading: A test of competing models of lexical ambiguity resolution. Journal of Memory \& Language, 31, 461-476.

Duffy, S. A., Henderson, J. M., \& Morris, R. K. (1989). Semantic facilitation of lexical access during sentence processing. Journal of Experimental Psychology: Learning, Memory, \& Cognition, 15, 791-801.

Duffy, S. A., Morris, R. K., \& RAYner, K. (1988). Lexical ambiguity and fixation times in reading. Journal of Memory \& Language, 27, 429-446.

Ferretti, T. R. (2000). Situation schemas, thematic roles and grammatical morphemes. Unpublished doctoral dissertation, University of Western Ontario.

Ferretti, T. R., McRae, K., \& Hatherell, A. (2001). Integrating verbs, situation schemas, and thematic role concepts. Journal of Memory \& Language, 44, 516-547.
Fodor, J. A. (1983). Modularity of mind. Cambridge, MA: MIT Press. Foss, D. J. (1982). A discourse on semantic priming. Cognitive Psychology, 14, 590-607.

Foss, D. J., \& SPEER, S. R. (1991). Global and local context effects in sentence processing. In R. R. Hoffman \& D. S. Palmero (Eds.), Cognition and the symbolic processes: Vol. III. Applied and ecological perspectives (pp. 115-139). Hillsdale, NJ: Erlbaum.

FrAncis, W. N., \& KuČERA, H. (1982). Frequency analysis of English usage: Lexicon and grammar. Boston: Houghton Mifflin.

Gough, P. B., Alford, J. A., Jr., \& Holley-Wilcox, P. (1981). Words and contexts. In O. J. L. Tzeng \& H. Singer (Eds.), Perception of print: Reading research in experimental psychology (pp. 85-102). Hillsdale, NJ: Erlbaum.

Greene, S. B., McKoon, G., \& Ratcliff, R. (1992). Pronoun resolution and discourse models. Journal of Experimental Psychology: Learning, Memory, \& Cognition, 18, 266-283.

Hess, D. J., Foss, D. J., \& Carroll, P. (1995). Effects of global and local context on lexical processing during language comprehension. Journal of Experimental Psychology: General, 124, 62-82.

Hogaboam, T. W., \& Perfetti, C. A. (1975). Lexical ambiguity and sentence comprehension. Journal of Verbal Learning \& Verbal Behavior, 14, 265-274.

Johnson-Laird, P. N. (1983). Mental models. Cambridge, MA: Harvard University Press.

Just, M. A., Carpenter, P. A., \& Woolley, J. D. (1982). Paradigms and processes in reading comprehension. Journal of Experimental Psychology: General, 111, 228-238.

KашАмото, A. Н. (1993). Nonlinear dynamics in the resolution of lexical ambiguity: A parallel distributed processing account. Journal of Memory \& Language, 32, 474-516.

Kellas, G., Paul, S. T., Martin, M., \& Simpson, G. B. (1991). Contextual feature activation and meaning access. In G. B. Simpson (Ed.), Understanding word and sentence (pp. 47-71). Amsterdam: Elsevier.

Kellas, G., \& VU, H. (1999). Strength of context does modulate the subordinate bias effect: A reply to Binder and Rayner. Psychonomic Bulletin \& Review, 6, 511-517.

Lancaster, J. S., \& Barsalou, L. W. (1997). Multiple organizations of events in memory. Memory, 5, 569-599.

MARSLEN-WiLSON, W. (1987). Functional parallelism in spoken wordrecognition. Cognition, 25, 71-102.

Martin, C., Vu, H., Kellas, G., \& Metcalf, K. (1999). Strength of discourse context as a determinant of the subordinate bias effect. Quarterly Journal of Experimental Psychology, 52A, 813-839.

McClelland, J. L. (1987). The case for interactionism in language processing. In M. Coltheart (Ed.), Attention and performance XII: The psychology of reading (pp. 3-36). Hillsdale, NJ: Erlbaum.

McClelland, J. L., St. John, M., \& Taraban, R. (1989). Sentence comprehension: A parallel distributed processing approach. Language \& Cognitive Processes, 4, 287-335.

McRae, K., Ferretti, T. R, \& Amyote, L. (1997). Thematic roles as verb-specific concepts. Language \& Cognitive Processes, 12, 137 176.

McRae, K., Hare, M., Ferretti, T. R., \& Elman, J. L. (2001). Activating verbs from typical agents, patients, instruments, and locations via event schemas. In J. D. Moore \& K. Stenning (Eds.), Proceedings of the Twenty-Third Annual Conference of the Cognitive Science Society (pp. 617-622). Mahwah, NJ: Erlbaum.

Nelson, D. L., McEvoy, C. L., WAlling, J. R. \& Wheeler, J. W., JR. (1980). The University of South Florida homograph norms. Behavior Research Methods \& Instrumentation, 12, 16-37.

O'Seaghdha, P. G. (1989). The dependence of lexical relatedness effects on syntactic connectedness. Journal of Experimental Psychology: Learning, Memory, \& Cognition, 15, 73-87.

Paul, S. T., Kellas, G., Martin, M., \& Clark, M. B. (1992). Influence of contextual features on the activation of ambiguous word meanings. Journal of Experimental Psychology: Learning, Memory, \& Cognition, 18, 703-717.

RAYNER, K., Binder, K. S., \& DufFy, S. A. (1999). Contextual strength and the subordinate bias effect: Comment on Martin, Vu, Kellas, and Metcalf. Quarterly Journal of Experimental Psychology, 52A, 841852. 
RAYNer, K., \& Frazier, L. (1989). Selection mechanisms in reading lexically ambiguous words. Journal of Experimental Psychology: Learning, Memory, \& Cognition, 15, 779-790.

Rayner, K., Pacht, J. M., \& Duffy, S. A. (1994). Effects of prior encounter and global discourse bias on the processing of lexically ambiguous words: Evidence from eye fixations. Journal of Memory \& Language, 33, 527-544.

RAYNER, K., \& WeLL, A. D. (1996). Effects of contextual constraint on eye movements in reading: A further examination. Psychonomic Bulletin \& Review, 3, 504-509.

SANFORd, A. J., \& GARRod, S. C. (1981). Understanding written language. New York: Wiley.

SCHANK, R. C., \& ABELSON, R. P. (1977). Scripts, plans, goals, and understanding. Hillsdale, NJ: Erlbaum.

Schwanenflugel, P. J., \& Shoben, E. J. (1985). The influence of sentence constraint on the scope of facilitation for upcoming words. Journal of Memory \& Language, 24, 232-252.

Sedivy, J. C., Tanenhaus, M. K., Chambers, C. G., \& Carlson, G. N. (1999). Achieving incremental semantic interpretation through contextual representation. Cognition, 71, 109-147.

Seidenberg, M. S., Tanenhaus, M. K., Leiman, J. M., \& BienKOWSKI, M. (1982). Automatic access of the meanings of ambiguous words in context: Some limitations of knowledge-based processing. Cognitive Psychology, 14, 489-537.

Simpson, G. B., \& KrUEgER, M. A. (1991). Selective access of homograph meanings in sentence context. Journal of Memory \& Language, 30, 627-643.

Simpson, G. B., Peterson, R. R., Casteel, M. A., \& Burgess, C. (1989). Lexical and sentence context effects in word recognition. Journal of Experimental Psychology: Learning, Memory, \& Cognition, 15, 88-97.

Solso, R. L., \& Juel, C. L. (1980). Positional frequency and versatility of bigrams for two- through nine-letter English words. Behavior Research Methods \& Instrumentation, 12, 297-343.

STANOVICH, K. E., \& WEST, R. F. (1983). On priming by a sentence context. Journal of Experimental Psychology: General, 112, 1-36.

SWINNEY, D. A. (1979). Lexical access during sentence comprehension: (Re)consideration of context effects. Journal of Verbal Learning \& Verbal Behavior, 18, 645-659.

TAвossi, P., Colombo, L., \& Job, R. (1987). Accessing lexical ambiguity: Effects of context and dominance. Psychological Research, 49, 161-167.

Taraban, R., \& McClelland, J. L. (1988). Constituent attachment and thematic role assignment in sentence processing: Influences of content-based expectations. Journal of Memory \& Language, 27, 597-632.

TraXler, M. J., \& Foss, D. J. (2000). Effects of sentence constraint on priming in natural language comprehension. Journal of Experimental Psychology: Learning, Memory, \& Cognition, 26, 1266-1282.

Traxler, M. J., Foss, D. J., Seely, R. E., Kaup, B., \& Morris, R. K. (2000). Priming in sentence processing: Intralexical spreading activation, schemas, and situation models. Journal of Psycholinguistic Research, 29, 581-595.

Twilley, L. C., Dixon, P., Tay lor, D., \& Clark, K. (1994). University of Alberta norms of relative meaning frequency for 566 homonyms. Memory \& Cognition, 22, 111-126.

Van Petten, C., Coulson, S., Rubin, S., Plante, E., \& Parks, M. (1999). Time course of word identification and semantic integration in spoken language. Journal of Experimental Psychology: Learning, Memory, \& Cognition, 25, 394-417.

Van Petten, C., \& Kutas, M. (1987). Ambiguous words in context: An event-related potential analysis of the time course of meaning activation. Journal of Memory \& Language, 26, 188-208.

Vu, H., \& Kellas, G. (1999). Contextual strength modulates the subordinate bias effect: Reply to Rayner, Binder, and Duffy. Quarterly Journal of Experimental Psychology, 52A, 853-856.

Vu, H., Kellas, G., \& Metcalf, K. (2000). The influence of thematic role fillers and agent filters [sic] likelihood on lexical ambiguity resolution. Abstracts of the Psychonomic Society, 5, 14.

Vu, H., Kellas, G., Metcalf, K., \& Herman, R. (2000). The influence of global discourse on lexical ambiguity resolution. Memory \& Cognition, 28, 236-252.
Vu, H., Kellas, G., \& Paul, S. T. (1998). Sources of sentence constraint on lexical ambiguity resolution. Memory \& Cognition, 26, 979-1001.

Whitney, P., Budd, D., Bramucci, R. S., \& Crane, R. S. (1995). On babies, bath water, and schemata: A reconsideration of top-down processes in comprehension. Discourse Processes, 20, 135-166.

WiLEY, J., \& RAYNER, K. (2000). Effects of titles on the processing of text and lexically ambiguous words: Evidence from eye movements. Memory \& Cognition, 28, 1011-1021.

ZwaAn, R. A., \& Radvansky, G. A. (1998). Situation models in language comprehension and memory. Psychological Bulletin, 123, 162-185.

\section{NOTES}

1. We use the general terms situation, situation structure, and situational representation, rather than the formal terms script and schema that are reserved for these theories (Bower et al., 1979; Schank \& Abelson, 1977). However, a situation can certainly be realized as a script or schema.

2. The research of Kellas and colleagues provides support for their contextual-feature-sensitive model of language processing, whereas the work of Rayner and colleagues provides evidence for their reorderedaccess model. There are several theoretical differences between the models, but the most fundamental difference concerns the fate of the dominant meaning when a polarized homonym (an ambiguous word with one highly dominant meaning) is processed in a subordinate biased context. For the Rayner group, a subordinate biased context will activate the subordinate meaning of a polarized ambiguous word, but it cannot preclude the dominant meaning from being activated. In contrast, according to the Kellas group, a strongly biased subordinate context can overwhelm the effect of meaning frequency, so that only the subordinate meaning will be activated. This critical difference has created quite a controversy, leading to several heated exchanges among the researchers for the past few years. It is not the intent of the present article to rekindle the controversy or argue the merits of each model. To do so would belabor an already well-debated issue and, thus, detract from the focus of the present research. We refer the reader to Binder and Rayner (1998, 1999) and Rayner, Binder, and Duffy (1999), as well as Kellas and Vu (1999), Martin et al. (1999), and Vu and Kellas (1999), for more elaborate discussions.

3. It is very likely that this fundamental debate will continue unabated and may never be resolved because, realistically, the selection-integration hypothesis can always be argued in lieu of meaning activation, since the temporal window for capturing multiple activation can always be shortened to preserve the principles of modularity (see also Carpenter, Miyake, \& Just, 1995).

4. Formally, this expression is part of the noun phrase of a prepositional phrase. Henceforth, we will use the term prepositional phrase noun filler, because it clearly refers to the word of interest (see Taraban \& McClelland, 1988).

5 . We thank one anonymous reviewer for providing precise descriptions and terminology that linked our off-line normative task with the on-line naming task in Experiment 1. As the reviewer will note, we adopted these descriptions almost verbatim, due to their clarity.

6. Due to a large number of cells with zeros for entries, a formal analysis of the error rates was not conducted. However, an inspection of Table 2 provides no evidence of a speed-accuracy tradeoff between error rates and latencies. This conclusion was supported by the Pearson's correlation coefficient comparing naming latencies with error rates $(r=+.37)$. Similarly, formal error analyses were not conducted for Experiment 2. However, as is shown in Table 4, there was no evidence for a speed-accuracy tradeoff $(r=+.24)$.

7. Indeed, for this example, the evoked domain of reference constrained noun filler completions, so that there was a cluster around money (bank, treasurer, coin, president, etc.) and another cluster around fraud (criminal, boss, agent, etc.).

8. We interpret our results within an interactive-activation framework that proposes that meaning computation is context dependent. However, other process models that assume fast integration following contextindependent multiple activation may be able to explain the data. Most notably, Duffy et al.'s (1988) reordered-access model and Traxler and 
Foss's (2000) facilitated integration model may explain the data if it is assumed that the 0 -msec ISI between sentence context and probe word is enough time for the processes of selection and integration, following context-independent activation, to occur. However, it was not our intent in this article to differentiate among these models. Our focus was to show that ambiguity resolution may be achieved via situational constraints and to examine the influence of a situation-evoking stimulus at different points in a linguistic stream.

9. Our model's processing assumption that the subordinate meaning of a polarized ambiguous word can be exclusively activated led one reviewer to question whether the model would predict that processing time for ambiguous words would be as short as that for unambiguous words in sufficiently strong contexts, since this would be contradictory to other research. In particular, Rayner and colleagues (e.g., Rayner et al., 1994) have consistently demonstrated that gaze durations for polarized ambiguous words in subordinate biased contexts are longer than those for unambiguous control words. It is our contention, however, that in none of these studies did the researchers assess the impact of contextual strength on the ambiguous word or the unambiguous control word. If the unambiguous controls fit better into their linguistic contexts than the ambiguous words into their contexts, control words should be processed more quickly than the ambiguous words. Second, the base level of activation of the subordinate meaning may be lower than that of an unambiguous word, and so more processing cycles may be needed for the activation level to reach threshold. Third, we would submit that even a contextually matched unambiguous control may still be processed more quickly than a polarized ambiguous word in a subordinate biased context, because an unambiguous word has no alternate at the meaning level that is competing for activation. In this situation, longer processing time on the ambiguous word does not necessitate the activation of multiple meanings. Finally, Martin et al. (1999) showed that processing time for a polarized ambiguous word in a strong subordinate biased context is equal to that for the same ambiguity in a strong dominant biased context. Since Rayner and colleagues have always demonstrated that processing time for an ambiguous word (polarized or balanced) in a dominant biased context is equal to that for an unambiguous control, we interpreted our results as demonstrating that a strong subordinate biased context can reduce processing time for a polarized ambiguous word (see, however, Binder \& Rayner, 1998, 1999).

(Manuscript received July 17, 2002; revision accepted for publication July 23, 2003.) 